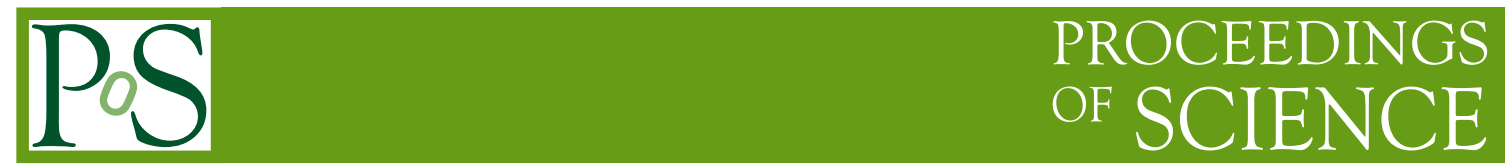

\title{
Review of Belle results
}

\section{Pavel Krokovny*}

Budker Institute of Nuclear Physics and Novosibirsk State University

E-mail: krokovny@inp.nsk.su

The Belle experiment, running at the $\mathrm{KEKB} e^{+} e^{-}$asymmetric energy collider during the first decade of the century, achieved its original objective of measuring precisely differences between particles and anti-particles in the $B$ system. After collecting $1000 \mathrm{fb}^{-1}$ of data at various $\Upsilon$ resonances, Belle also obtained the many other physics results described in this article.

The XXI International Workshop High Energy Physics and Quantum Field Theory,

June 23 âĂS June 30, 2013

Saint Petersburg Area, Russia

${ }^{*}$ Speaker. 


\section{Introduction}

In the sections that follow, we describe the physics accomplishments of the Belle experiment [1], which ran at the KEKB [2] $e^{+} e^{-}$asymmetric energy collider in Tsukuba, Japan between 1999 and 2010. KEKB broke all records for integrated and instantaneous luminosity for a high energy accelerator. As a result Belle was able to integrate over $1000 \mathrm{fb}^{-1}$ or one inverse attobarn of data. Most of the Belle luminosity was recorded on or near the $\Upsilon(4 S)$ resonance, which is the optimal center of mass (CM) energy for the production of $B \bar{B}$ pairs used in $B$ physics analysis.

Neutral $B$ mesons, $B^{0}$ and $\bar{B}^{0}$, can transform or mix into their antiparticles through box diagrams. The frequency of the mixing transition (oscillation) is $\Delta m_{d}=(0.507 \pm 0.004) \mathrm{ps}^{-1}$ [3], while the lifetime $\left(\tau_{B^{0}}\right)$ is $1.519 \pm 0.007 \mathrm{ps}$ [河].

At a $B$-factory, pairs of neutral $B$ mesons in a coherent state with $C=-1$ are produced by $\Upsilon(4 S) \rightarrow B^{0} \bar{B}^{0}$ decays In a decay in which one $B$ meson decays to $f_{C P}$ and the other $B$ meson decays to a flavor specific final state, $f_{\text {tag }}$, the decay rate is given as

$$
\mathscr{P}(\Delta t, q ; \mathscr{S}, \mathscr{A})=\frac{e^{-|\Delta t| / \tau_{B^{0}}}}{4 \tau_{B^{0}}}\left\{1+q \cdot\left[\mathscr{S} \sin \left(\Delta m_{d} \Delta t\right)+\mathscr{A} \cos \left(\Delta m_{d} \Delta t\right)\right]\right\} .
$$

Here $\Delta t=t_{C P}-t_{\text {tag }}$ is the difference between the proper decay times of $f_{C P}$ and $f_{\text {tag }}, q= \pm 1$ is the flavor of $f_{\text {tag }}\left(+1\right.$ for $B^{0} \rightarrow f_{\text {tag }}$ ). The quantities $\mathscr{S}$ and $\mathscr{A}$ are $C P$ violation parameters that are dependent on the decay mode. The parameter $\mathscr{S}$ describes mixing-induced $C P$ violation and is given by $\mathscr{S}=-\eta_{C P} \sin 2\left(\phi_{1}-\phi_{D}\right)$, where $\eta_{C P}$ is the $C P$ eigenvalue of $f_{C P}$. The other parameter, $\mathscr{A}$, corresponds to direct $C P$ violation (i.e. no $C P$ violation in the $B^{0} \leftrightarrow \bar{B}^{0}$ transition rates). It should be noted that, depending on the weak phase of the decay, $C P$ violation measurements give information on the various angles of the unitarity triangle. The asymmetry in the rate of $B^{0}$ and $\bar{B}^{0}$ decays is given by

$$
A(\Delta t) \equiv \frac{\mathscr{P}(\Delta t,+1 ; \mathscr{S}, \mathscr{A})-\mathscr{P}(\Delta t,-1 ; \mathscr{S}, \mathscr{A})}{\mathscr{P}(\Delta t,+1 ; \mathscr{S}, \mathscr{A})+\mathscr{P}(\Delta t,-1 ; \mathscr{S}, \mathscr{A})}=\mathscr{S} \sin \Delta m_{d} \Delta t+\mathscr{A} \cos \Delta m_{d} \Delta t
$$

An experimental measurement of time-dependent $C P$ violation at a $B$-factory includes the following steps: reconstruct one $B$ decaying to $f_{C P}$; determine $q$ using all available information on the $B \rightarrow f_{\text {tag }}$ decay; reconstruct vertices for $f_{C P}$ and $f_{\text {tag }}$ and determine $\Delta t$ from the distance between the two $B$ vertices; obtain $\mathscr{S}$ and $\mathscr{A}$ by fitting the $\Delta t$ distribution of reconstructed signal candidates.

\section{Measurement of $\phi_{1}$}

At the quark level neutral $B$ meson decays into $(c \bar{c}) K^{0}$ are induced by a $b \rightarrow c \bar{c} s$ transition. Since both leading and sub-leading order diagrams of this process contain neither $V_{u b}$ nor $V_{t d}$, there is no complex phase in the decay amplitude. Thus $\phi_{D}$ is zero and the mixing-induced $C P$ violation parameter $\mathscr{S}$ is directly related to one of the $C P$-violating angles, $\phi_{1}$. In the $\mathrm{SM}, \mathscr{S}=-\eta_{C P} \cdot \sin 2 \phi_{1}$ and $\mathscr{A} \approx 0$ are expected. 


\section{$2.1 B^{0} \rightarrow(c \bar{c}) K^{0}$ reconstruction}

We reconstruct $J / \psi K_{S}^{0}, J / \psi K_{L}^{0}, \psi(2 S) K_{S}^{0}$, and $\chi_{c 1} K_{S}^{0}$ as the $f_{C P}$ in neutral $B$ meson decays to $(c \bar{c}) K^{0} . J / \psi$ mesons are reconstructed via their decay into oppositely charged lepton pairs $\left(e^{+} e^{-}\right.$ or $\mu^{+} \mu^{-}$) while $\psi(2 S)$ mesons are reconstructed by lepton pairs as well as $J / \psi \pi^{+} \pi^{-}$final states. We reconstruct $\chi_{c 1}$ mesons in the $J / \psi \gamma$ mode and $K_{S}^{0}$ mesons in the $\pi^{+} \pi^{-}$final state.

For $B^{0} \rightarrow J / \psi K_{S}^{0}, \psi(2 S) K_{S}^{0}$, and $\chi_{c 1} K_{S}^{0}$ candidates, the $B$ signal is identified using two kinematic variables calculated in the $\Upsilon(4 S) \mathrm{CM}$ : the energy difference $\Delta E \equiv E_{B}^{*}-E_{\text {beam }}^{*}$ and the beamenergy constrained mass $M_{\mathrm{bc}} \equiv \sqrt{\left(E_{\text {beam }}^{*}\right)^{2}-\left(p_{B}^{*}\right)^{2}}$, where $E_{\text {beam }}^{*}$ is the beam energy in the CM of the $\Upsilon(4 S)$ resonance, and $E_{B}^{*}$ and $p_{B}^{*}$ are the $\mathrm{CM}$ energy and momentum of the reconstructed $B$ candidate, respectively. In the $B^{0} \rightarrow J / \psi K_{L}^{0}$ case, candidate $K_{L}^{0}$ mesons are selected using information recorded in the ECL and/or the KLM. Since the $K_{L}^{0}$ energy cannot be measured, we determine only its direction. Thus $B^{0} \rightarrow J / \psi K_{L}^{0}$ candidates are identified by the value of $p_{B}^{*}$ calculated using a two-body decay kinematic assumption.

The $M_{\mathrm{bc}}$ distribution for signal candidates with a stringent $\Delta E$ requirement $(|\Delta E|<40 \mathrm{MeV}$ for $J / \psi K_{S}^{0},|\Delta E|<30 \mathrm{MeV}$ for $\psi(2 S) K_{S}^{0}$, and $|\Delta E|<25 \mathrm{MeV}$ for $\left.\chi_{c 1} K_{S}^{0}\right)$ as well as the $p_{B}^{*}$ distribution for $J / \psi K_{L}^{0}$ candidates are shown in Fig. 1. The signal yields and purities are estimated for each $f_{C P}$ mode and given in Table 1 .
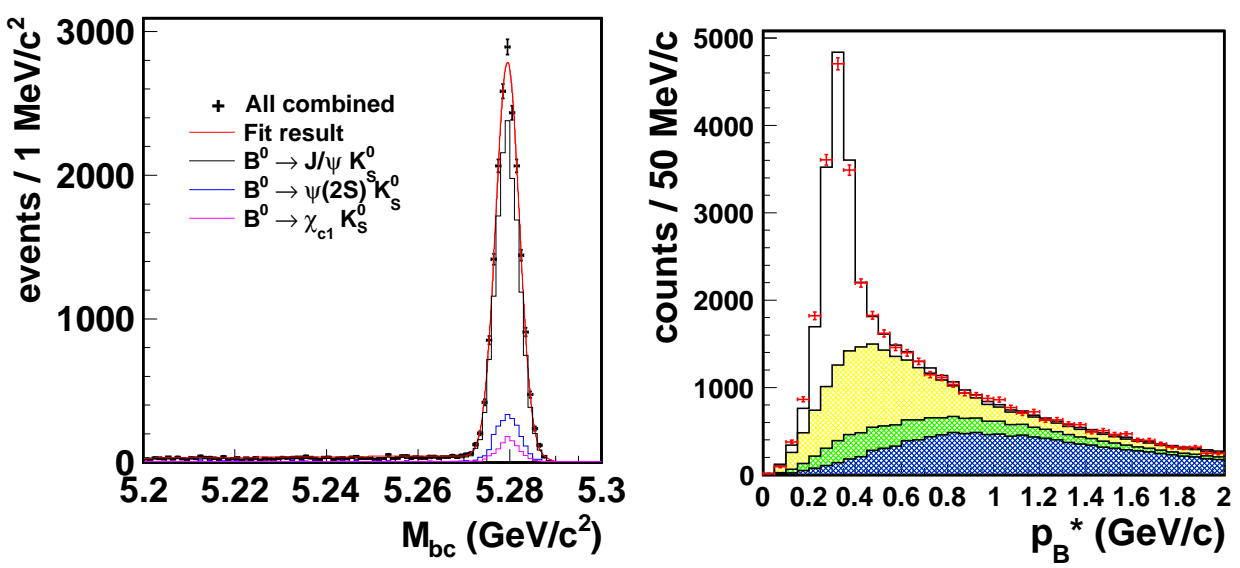

Figure 1: $M_{\mathrm{bc}}$ distribution within the $\Delta E$ signal region for $B^{0} \rightarrow J / \psi K_{S}^{0}$ (black), $\psi(2 S) K_{S}^{0}$ (blue), and $\chi_{c 1} K_{S}^{0}$ (magenta); the superimposed curve (red) shows the fit result for all modes combined (left) and the $p_{B}^{*}$ distribution for $B^{0} \rightarrow J / \psi K_{L}^{0}$ candidates with the results of the fit separately shown as signal (open histogram), background with a real $J / \psi$ and a real $K_{L}^{0}$ (yellow), background with a real $J / \psi$ but without a real $K_{L}^{0}$ (green), and background without a real $J / \psi$ (blue) (right).

\subsection{Flavor tagging}

For the events in which we reconstructed $B^{0} \rightarrow f_{C P}$ candidates, the neutral $B$ flavor is identified from the decay products of the accompanying $B$ meson. The available information is obtained from leptons, kaons, $\Lambda$ baryons, and pions. Leptons directly coming from $B$ decay and secondary leptons and strange particles in the cascade decays carry the mother $b$-flavor information. Low momentum 
Table 1: Signal yield $\left(N_{\text {sig }}\right), C P$ eigenvalue $\left(\eta_{C P}\right)$, and purity for each $B^{0} \rightarrow f_{C P}$ mode.

\begin{tabular}{lccc}
\hline \hline$B$ decay mode & $\eta_{C P}$ & $N_{\text {sig }}$ & Purity (\%) \\
\hline$J / \psi K_{S}^{0}$ & -1 & $12649 \pm 114$ & 97 \\
$\psi(2 S)\left(\ell^{+} \ell^{-}\right) K_{S}^{0}$ & -1 & $904 \pm 31$ & 92 \\
$\psi(2 S)\left(J / \psi \pi^{+} \pi^{-}\right) K_{S}^{0}$ & -1 & $1067 \pm 33$ & 90 \\
$\chi_{c 1} K_{S}^{0}$ & -1 & $940 \pm 33$ & 86 \\
$J / \psi K_{L}^{0}$ & +1 & $10040 \pm 154$ & 63 \\
\hline \hline
\end{tabular}

tagging pions may come from $D^{* \pm}$ decays. In addition, there are high momentum pions originating from $\bar{B}^{0} \rightarrow D^{(*)+} \pi^{-}$or $D^{(*)^{+}} \rho^{-}$decays. Both types of tagging pions give some information about $b$-flavor. The information from all the decay products is handled by a multi-dimensional likelihood approach with corresponding look-up tables.

To calibrate $w$, we select a flavor specific final state of neutral $B$ meson decays such as semileptonic $\overline{B^{0}} \rightarrow D^{*+} \ell^{-} \bar{v}$ decays and hadronic $\overline{B^{0}} \rightarrow D^{(*+)} \pi^{-}$and $D^{+*} \rho^{-}$decays. We then determine the wrong tag fraction $w$ by measuring the time evolution of the opposite-sign flavor asymmetry, as it exhibits a $\Delta t$ dependence proportional to $(1-2 w) \cos \left(\Delta m_{d} \Delta t\right)$. We also determine $\Delta w$, which is the difference in $w$ between $q=+1$ and -1 events. For $B^{0} \rightarrow J / \psi K_{S}^{0}$ decay, we obtain the effective tagging efficiency, $\varepsilon_{\text {eff }}=\varepsilon(1-2 w)^{2}=(30.1 \pm 0.4) \%$, where $\varepsilon$ is the tagging efficiency.

\section{3 $\Delta t$ determination and its resolution}

In energy-asymmetric $e^{+} e^{-}$collisions at KEKB, the $\Upsilon(4 S)$ is produced with a Lorentz boost of $\beta \gamma=0.425$ nearly along the $z$-axis, which is defined as the direction anti-parallel to the $e^{+}$beam at Belle. Since $B$ mesons are approximately at rest with respect to the $\Upsilon(4 S)$, we can measure $\Delta t$ by measuring the displacement between the two $B$ meson decay vertices in the $z$ direction, $\Delta z$, $\Delta t \simeq \frac{\Delta z}{\beta \gamma c}$.

The $B$ meson decay vertex is reconstructed by a Lagrange multiplier approach, which minimizes the $\chi^{2}$ calculated from the decay vertex position and the daughter particle tracks. We call this procedure a "vertex fit". The vertex fit is carried out using daughter tracks with a sufficient (minimal) number of SVD hits and a constraint on the interaction-region profile in the plane perpendicular to the beam axis.

Because of the negligible flight length of $J / \psi$ or $\psi(2 S)$ mesons, the vertex reconstructed from their daughter lepton tracks can represent the $B^{0} \rightarrow f_{C P}$ decay vertex; its resolution is found to be approximately $75 \mu \mathrm{m}$. On the other hand, the $B^{0} \rightarrow f_{\text {tag }}$ vertex is obtained with well-reconstructed tracks that are not assigned to $f_{C P}$. Here, high momentum leptons are always retained because they usually come directly from semileptonic $B$ meson decays. Since $f_{\text {tag }}$ may contain long-lived particles such as $D^{+}, D^{0}, K_{S}^{0}$, and so on, the vertex reconstructed using the daughter tracks coming from these intermediate particles can deviate from the true $B^{0} \rightarrow f_{\text {tag }}$ vertex. This effect is minimized by removing tracks that are identified by a large contribution to the vertex fit $\chi^{2}$. The $f_{\text {tag }}$ vertex position resolution is found to be approximately $165 \mu \mathrm{m}$. 
In the Belle experiment, the contributions to $\Delta t$ measurement error are divided into three categories: detector measurement error, the effect of secondary particles in $f_{\text {tag }}$ vertex reconstruction, and the kinematical approximation, $\Delta t \simeq \Delta z /(\beta \gamma c)$. These three effects are convoluted on an eventby-event basis to obtain the $\Delta t$ resolution function, which is used in a maximum likelihood fit to extract $\mathscr{S}$ and $\mathscr{A}$ as discussed in the next section.

\subsection{Extracting $C P$ violation parameters}

We determine $\sin 2 \phi_{1}$ and $\mathscr{A}$ from a maximum likelihood fit using $\Delta t$ and $q$ information obtained on an event-by-event basis from signal candidates. By taking the effect of incorrect flavor assignment into account, the probability density function (PDF) expected for the signal distribution is given by

$$
\mathscr{P}_{\text {sig }}(\Delta t)=\frac{e^{-|\Delta t| / \tau_{B^{0}}}}{4 \tau_{B^{0}}}\left\{1-q \Delta w_{l}+q\left(1-2 w_{l}\right) \times\left[\left(-\eta_{C P}\right) \sin 2 \phi_{1} \sin \left(\Delta m_{d} \Delta t\right)+\mathscr{A} \cos \left(\Delta m_{d} \Delta t\right)\right]\right\} .
$$

The distribution is convoluted with the $\Delta t$ resolution function $R_{\mathrm{sig}}(\Delta t)$, which takes into account the finite vertex resolution as described in Sect. 2.3. The background PDF $\mathscr{P}_{\mathrm{bkg}}(\Delta t)$ is determined by the events found in a sideband region well away from the signal region in $M_{\mathrm{bc}}-\Delta E$ space as well as Monte Carlo (MC) events. A small component of broad outliers in the $\Delta z$ distribution, caused by misreconstruction, is represented by a Gaussian function $\mathscr{P}_{\mathrm{ol}}(\Delta t)$ with $\sigma \approx 30 \mathrm{ps}$. We determine the following likelihood value for each event indexed by $i$ :

$$
\mathscr{P}_{i}\left(\Delta t_{i}, q_{i} ; \sin 2 \phi_{1}, \mathscr{A}\right)=\left(1-f_{\mathrm{ol}}\right) f_{\mathrm{sig}} \int_{-\infty}^{\infty} \mathscr{P}_{\mathrm{sig}}\left(\Delta t^{\prime}\right) R_{\mathrm{sig}}\left(\Delta t_{i}-\Delta t^{\prime}\right) d\left(\Delta t^{\prime}\right)+\left(1-f_{\mathrm{ol}}\right) f_{\mathrm{bkg}} \mathscr{P}_{\mathrm{bkg}}\left(\Delta t_{i}\right)+f_{\mathrm{ol}} P_{\mathrm{ol}}\left(\Delta t_{i}\right),
$$

where $f_{\mathrm{ol}}$ is the outlier fraction, $f_{\text {sig }}$ and $f_{\mathrm{bkg}}$ are the signal and background probabilities calculated as functions of $\Delta E$ and $M_{\mathrm{bc}}$. The $C P$ violation parameters, $\sin 2 \phi_{1}$ and $\mathscr{A}$, are determined by maximizing the likelihood function $L\left(\sin 2 \phi_{1}, \mathscr{A}\right)=\prod_{i} \mathscr{P}_{i}\left(\Delta t_{i}, q_{i} ; \sin 2 \phi_{1}, \mathscr{A}\right)$, where the product runs over all events. A fit to the candidate events results in the $C P$ violation parameters 㺻,

$$
\begin{aligned}
\sin 2 \phi_{1} & =0.667 \pm 0.023 \text { (stat) } \pm 0.012 \text { (syst) } \\
\mathscr{A} & =0.006 \pm 0.016 \text { (stat) } \pm 0.012 \text { (syst). }
\end{aligned}
$$

The background-subtracted $\Delta t$ distribution for $q=+1$ and $q=-1$ events and the asymmetry for events with good quality tags are shown in Fig. 2. The world average of $\sin 2 \phi_{1}$ is now $0.68 \pm 0.02$, which is a firm SM reference.

\subsection{Search for new physics using $C P$ violation measurements in $b \rightarrow s$ penguin modes.}

$B$ meson decays involving penguin diagrams are thought to be a sensitive probe for new physics (NP) beyond the SM because of the one-loop nature of penguins. NP could appear as deviations of $C P$ violation parameters from the SM expectation. In this section, some highlight results for penguin modes are reviewed.

In SM $b \rightarrow s \bar{q} q$ hadronic $B$ decays, the relevant coupling is $V_{t b}^{*} V_{t s}$ and the weak phase is the same as in the $b \rightarrow c \bar{c} s$ transition, e.g. $B^{0} \rightarrow(c \bar{c}) K^{0}$ decay. Therefore, the main point is to check whether the penguin $C P$ violation results deviate from the SM expectation, $\mathscr{S}=-\eta_{C P} \sin 2 \phi_{1}$ and 

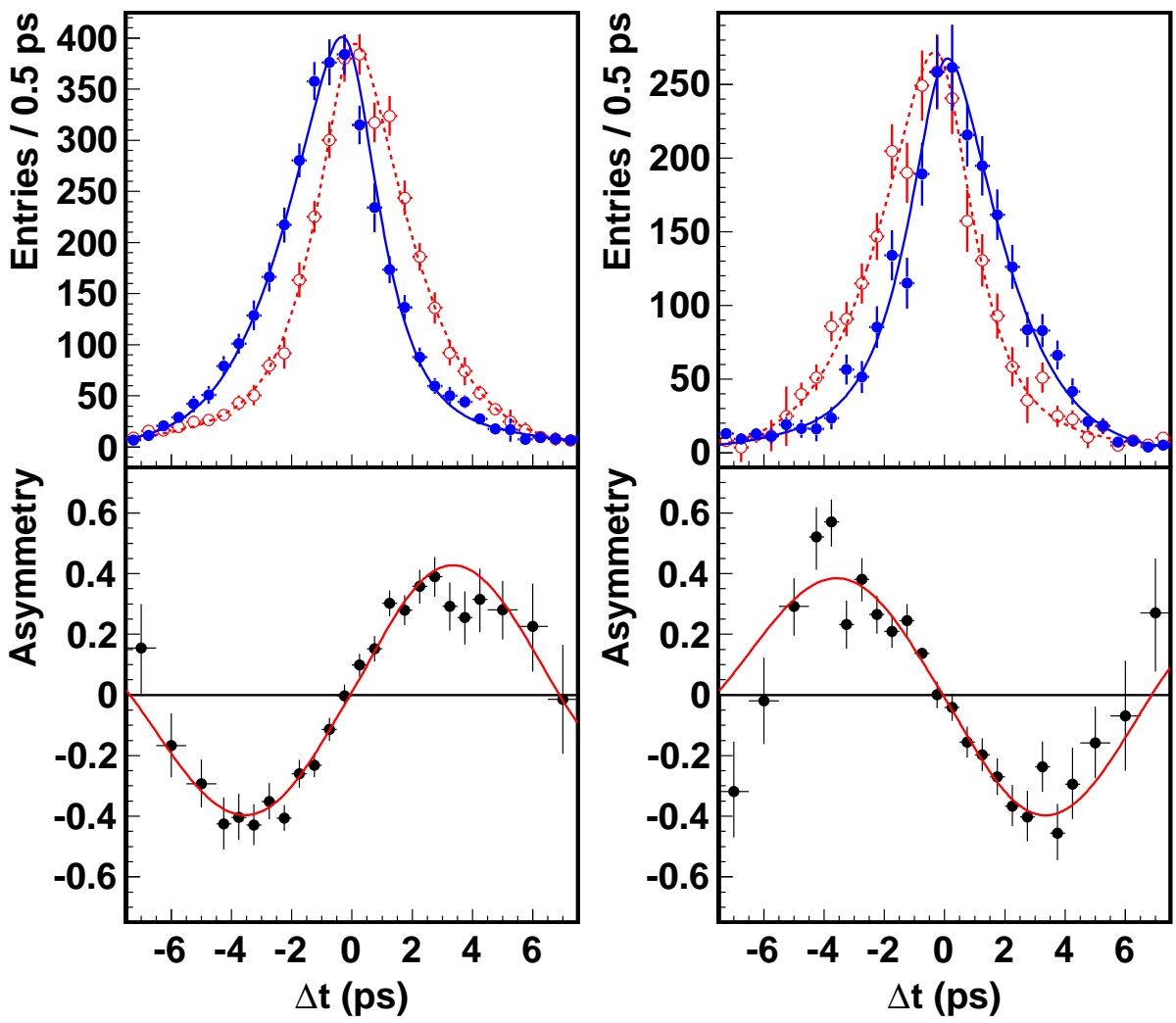

Figure 2: The background-subtracted $\Delta t$ distribution for $q=+1$ (red) and $q=-1$ (blue) events and asymmetry for events with good quality tags in $(c \bar{c}) K_{S}^{0}$ (left) and $J / \psi K_{L}^{0}$ (right) decays.

$\mathscr{A}=0$. In this context, the time-dependent $C P$-violating parameters are denoted as $\sin 2 \phi_{1}^{\text {eff }}$ and $\mathscr{A}$. The modes $B^{0} \rightarrow \phi K^{0}, \eta^{\prime} K^{0}$, and $K^{0} K^{0} K^{0}$ that involve only $b \rightarrow s \bar{s}$ processes are of special interest, since the SM theoretical uncertainty for $C P$ violation is small for these decay processes.

In the Belle experiment, attempts to perform measurements of time-dependent $C P$ violation in $b \rightarrow s \bar{q} q$ induced decays with $B^{0} \rightarrow \eta^{\prime} K_{S}^{0}$ and $\phi K_{S}^{0}$ modes were made from the earliest stage of data taking, starting in 2002. In 2003, using a $152 \times 10^{6} B \bar{B}$ data sample, the value of $\mathscr{S}$ in $B^{0} \rightarrow \phi K_{S}^{0}$ flipped sign and exhibited a $3.5 \sigma$ deviation from the $\mathrm{S}$ parameter measured in $B^{0} \rightarrow$ $(c \bar{c}) K^{0}$ modes [f]. This was very striking and suggestive of an NP effect. In 2006, with a larger statistics data sample corresponding to $535 \times 10^{6} B \bar{B}$, updated measurements were reported. These measurements added $B^{0} \rightarrow \eta^{\prime} K_{L}^{0}$ and $\phi K_{L}^{0}$ decays to the $B^{0} \rightarrow \eta^{\prime} K^{0}$ and $\phi K^{0}$ sample [6]. The results are summarized in Table 2. In $B^{0} \rightarrow \eta^{\prime} K^{0}$ decay, $C P$ violation is observed with a statistical significance of 5.6 $\sigma$. In all these three $B$ decay modes, the large deviation from $B^{0} \rightarrow(c \bar{c}) K^{0}$ has disappeared.

In spite of the small theoretical uncertainty, experimentally, several contributions overlap in $B^{0} \rightarrow \phi K^{0}$ because of the relatively wide natural widths of the resonances that contribute in the 
Table 2: Measurements of $C P$ violation parameters, $\sin 2 \phi_{1}^{\text {eff }}$ and $\mathscr{A}$, in $B^{0} \rightarrow \eta^{\prime} K^{0}$, $\phi K^{0}$, and $K_{S}^{0} K_{S}^{0} K_{S}^{0}$ modes with a $535 \times 10^{6} B \bar{B}$ data sample. The first and second errors are statistical and systematic errors, respectively.

\begin{tabular}{lcc}
\hline \hline$B$ decay mode & $\sin 2 \phi_{1}^{\text {eff }}$ & $\mathscr{A}$ \\
\hline$\eta^{\prime} K^{0}$ & $+0.64 \pm 0.10 \pm 0.04$ & $-0.01 \pm 0.07 \pm 0.05$ \\
$\phi K^{0}$ & $+0.50 \pm 0.21 \pm 0.06$ & $+0.07 \pm 0.15 \pm 0.05$ \\
$K_{S}^{0} K_{S}^{0} K_{S}^{0}$ & $+0.30 \pm 0.32 \pm 0.08$ & $-0.31 \pm 0.20 \pm 0.07$ \\
\hline \hline
\end{tabular}

$K^{+} K^{-}$final state. In order to resolve these interfering contributions, Belle fits the time-dependent Dalitz distribution by expressing each contribution at the amplitude level for the $B^{0} \rightarrow K^{+} K^{-} K_{S}^{0}$ candidate events. With this technique, the extracted parameter is not $\sin 2 \phi_{1}^{\text {eff }}$ but rather the angle $\phi_{1}^{\text {eff }}$ itself and $\mathscr{A}$. Therefore the result does not have a two-fold ambiguity between $\phi_{1}^{\text {eff }}$ and $\pi / 2-\phi_{1}^{\text {eff }}$. In $B^{0} \rightarrow K^{+} K^{-} K_{S}^{0}$ decays, we find four solutions related to resonant amplitude interference. The preferred one is identified using external information related to $f_{0}(980)$ and $f_{X}$ (assumed to be $f_{0}(1500)$ ) branching fractions. The obtained $C P$ violation parameters are summarized in Table 3 团. These are consistent with the $C P$ violation in $B^{0} \rightarrow c \bar{c} K^{0}$ decays at the $1 \sigma$

Table 3: $C P$ violation parameters in $B^{0} \rightarrow K^{+} K^{-} K_{S}^{0}$ time-dependent Dalitz analysis, $\phi_{1}^{\text {eff }}$ and $\mathscr{A}$. The first, second, and third errors are statistical, experimental systematic, and Dalitz model uncertainties, respectively.

\begin{tabular}{lcc}
\hline \hline$B$ decay mode & $\phi_{1}^{\text {eff }}$ & $\mathscr{A}$ \\
\hline$\phi K_{S}^{0}$ & $(32.2 \pm 9.0 \pm 2.6 \pm 1.4)^{\circ}$ & $+0.04 \pm 0.20 \pm 0.10 \pm 0.02$ \\
$f_{0} K_{S}^{0}$ & $(31.3 \pm 9.0 \pm 3.4 \pm 4.0)^{\circ}$ & $-0.30 \pm 0.29 \pm 0.11 \pm 0.09$ \\
\hline \hline
\end{tabular}

level.

Including other $b \rightarrow s$ mediated $B$ decays, the precision of $\sin 2 \phi_{1}^{\text {eff }}$ is still statistically limited, typically $0.1 \sim 0.2$. Obtaining $\mathscr{O}\left(10^{-2}\right)$ sensitivity requires an integrated luminosity of $\mathscr{O}\left(10 \mathrm{ab}^{-1}\right)$, and a Super $B$-factory experiment.

\section{Measurement of $\phi_{2}$}

After the first observation of $C P$ violation in $B$ meson decays, which gave a measurement of $\phi_{1}$, a precise measurement of $\phi_{2}$ became the next target of $C P$ violation measurements for the validation of the Kobayashi-Maskawa model. The first Belle measurement of $C P$ asymmetry parameters in $B^{0} \rightarrow \pi^{+} \pi^{-}$decay [8] was reported in March 2002, representing the second decay mode (after $B \rightarrow c \bar{c} K^{0}$ ) with a time-dependent $C P$ violation measurement.

The decay modes used for $\phi_{2}$ measurements are those proceeding via $b \rightarrow u$ transition, such as $B^{0} \rightarrow \pi^{+} \pi^{-}, B^{0} \rightarrow \rho^{+} \pi^{-}, B^{0} \rightarrow \rho^{+} \rho^{-}$. The $b \rightarrow u$ transition is shown in Fig. B(left) and includes the Cabibbo-Kobayashi-Maskawa (CKM) element, $V_{u b}$; it can be shown that the time dependent $C P$ asymmetry is then given as $\mathscr{S}=\sin 2 \phi_{2}$ and $\mathscr{A} \simeq 0$. However, an additional amplitude, a 
"penguin diagram" (Fig. B (right)), contributes and has a phase that is different from the tree diagram $\left(V_{t d}\right.$ instead of $\left.V_{u b}\right)$. This causes a deviation of $\mathscr{S}$ from $\sin 2 \phi_{2}$ and a non-zero $\mathscr{A}$.

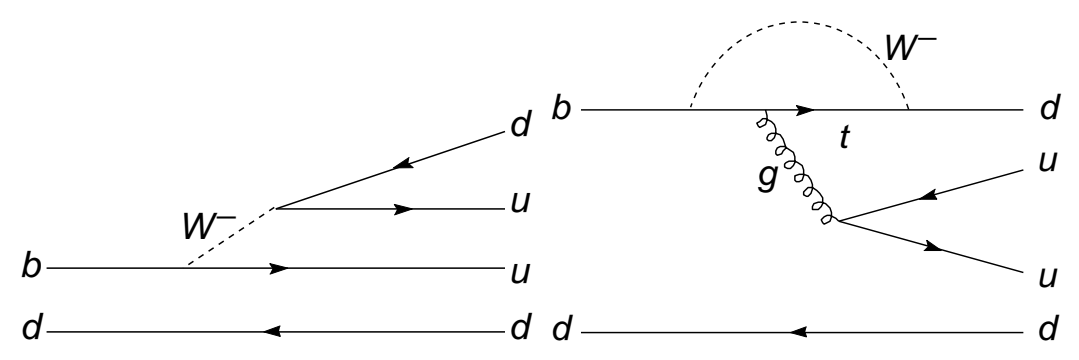

Figure 3: Tree (left) and penguin (right) diagrams for $B^{0} \rightarrow \pi^{+} \pi^{-}$decay.

The first $\phi_{2}$ measurement was attempted using the $B^{0} \rightarrow \pi^{+} \pi^{-}$decay mode. This decay has the simplest two-body topology and was one of the first well established charmless $B$ decays. The reconstruction of the decay is straightforward: a pair of oppositely charged pions with an invariant mass consistent with the $B$-meson mass $\left(M_{\mathrm{bc}}=m_{B}\right)$ is selected; the $B$ meson energy in CM is required to be consistent with the beam energy $(\Delta E=0)$. However, the selected sample suffers from a very large background from the $e^{+} e^{-} \rightarrow q \bar{q}(q=u, d, s, c)$ continuum process since the same kinematic properties can easily be faked by two oppositely charged pions fragmented from primary quarks and carrying about half of their momentum. Another significant background is from $B^{0} \rightarrow K^{+} \pi^{-}$decay, where the kaon is misidentified as a pion. The branching fraction for the former decay mode is about four times higher than that of $B^{0} \rightarrow \pi^{+} \pi^{-}$. In this case, the reconstructed $\Delta E$ is shifted by $-40 \mathrm{MeV}$ and good $K / \pi$ separation and good momentum resolution are important to reduce this background.

The continuum background is suppressed utilizing a difference in the global event topology for the two classes of events; continuum events have a two-jet like shape while $B \bar{B}$ events have an isotropic shape as the two $B$ mesons are produced almost at rest in the CM. To quantify the event shape, we use a Fisher discriminant combining modified Fox-Wolfram moments. We form a likelihood $\mathscr{L}_{s}\left(\mathscr{L}_{b}\right)$ for signal (continuum background) using the Fisher discriminant and the angle between the flight direction of the $B$ candidate and the beam direction in the $\mathrm{CM}, \cos \theta_{B}$. The likelihood ratio $\mathscr{R}=\mathscr{L}_{s} /\left(\mathscr{L}_{s}+\mathscr{L}_{b}\right)$ is used as the final continuum suppression parameter. In the early analyses [ [ $\mathbb{B}$, we imposed a tight requirement on $\mathscr{R}$ by optimizing $S / \sqrt{S+B}$, where $S$ and $B$ are the expected number of signal and background events, respectively. In a later analysis [9], we optimized the $\mathscr{R}$ requirement depending on the flavor tagging quality.

The $\Delta E$ distribution of $B^{0} \rightarrow \pi^{+} \pi^{-}$candidates is shown in Fig. 6 . Background events due to three-body decays populate the negative $\Delta E$ region but they do not contribute in the $B^{0} \rightarrow \pi^{+} \pi^{-}$ signal region $(|\Delta E|<0.064 \mathrm{GeV})$.

The vertex reconstruction and the flavor tagging are performed in the same way as for the $\sin 2 \phi_{1}$ measurements. The $C P$ violation parameters are extracted from a fit to the $\Delta t$ distribution for the events in the signal region in $\Delta E$ and $M_{\mathrm{bc}}\left([5.271,5.287] \mathrm{GeV} / \mathrm{c}^{2}\right)$. The PDFs include the signal, continuum background, and $B^{0} \rightarrow K^{+} \pi^{-}$background. The first result was reported using 

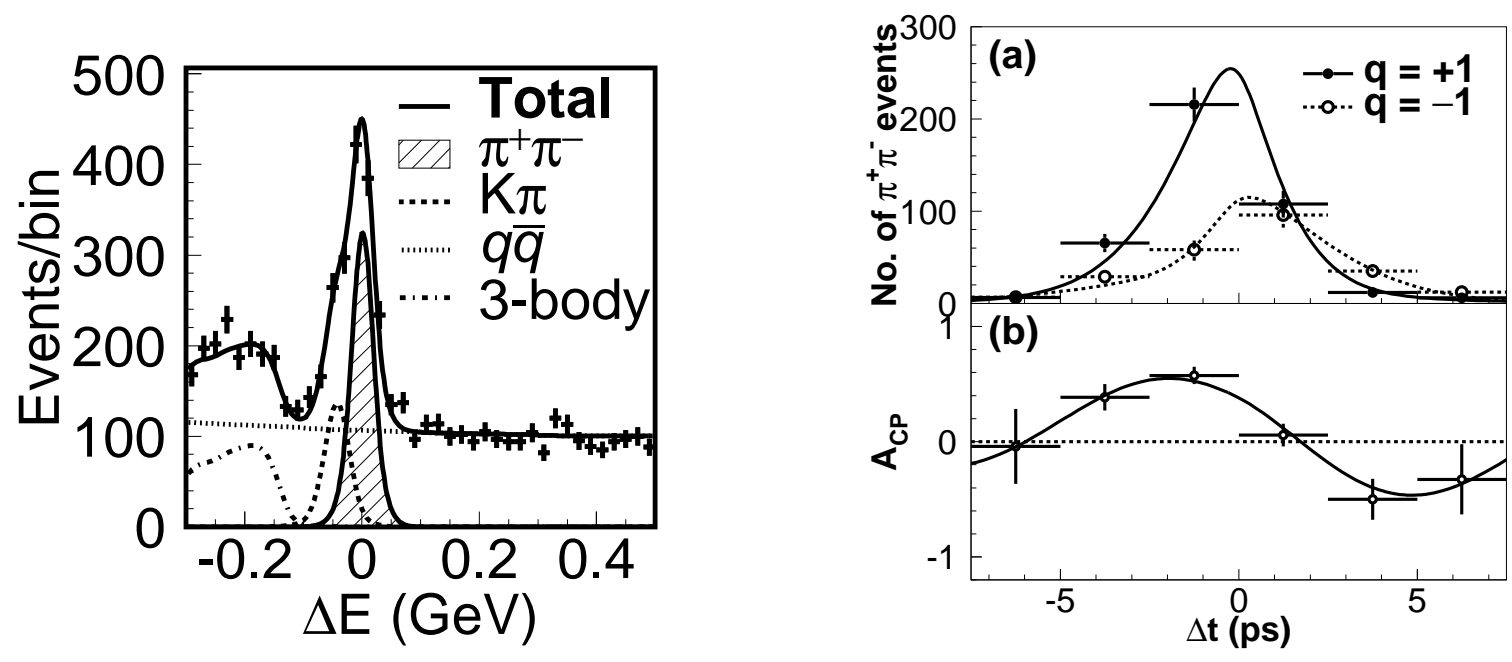

Figure 4: Left: $\Delta E$ distribution of $B^{0} \rightarrow \pi^{+} \pi^{-}$candidates. In order to enhance the signal, requirements are imposed on the two other variables, $M_{\mathrm{bc}}$ and $\mathscr{R}$. Right: $\Delta t$ distribution for $B^{0}$ and $\bar{B}^{0}$ tagged $B \rightarrow \pi^{+} \pi^{-}$ events (top) and the $C P$ asymmetry together with the fit result (bottom).

$48 \times 10^{6} B \bar{B}$ pairs: [8]

$$
\begin{array}{r}
\mathscr{S}_{\pi \pi}=-1.21_{-0.27}^{+0.38}(\text { stat })_{-0.13}^{+0.16}(\text { syst }) \\
\mathscr{A}_{\pi \pi}=+0.94_{-0.31}^{+0.25}(\text { stat }) \pm 0.09(\text { syst })
\end{array}
$$

In the latest analysis using $535 \times 10^{6} B \bar{B}$ pairs [9], a stringent selection on $K / \pi$ particle identification is not imposed and instead the $B^{0} \rightarrow K^{+} \pi^{-}$decays are included as a component in the fit to extract the $C P$ violation parameters. This increases the signal detection efficiency by $23 \%$ and improves the measurement errors by $10 \%$. The results are [9]

$$
\begin{aligned}
& \mathscr{S}_{\pi \pi}=-0.61 \pm 0.10(\text { stat }) \pm 0.04(\text { syst }) \\
& \mathscr{A}_{\pi \pi}=+0.55 \pm 0.08(\text { stat }) \pm 0.05(\text { syst }) .
\end{aligned}
$$

The $\Delta t$ distribution and the asymmetry together with fit results are shown in Fig. $甘$. A clear non-zero $\mathscr{A}_{\pi \pi}$, i.e. a clear direct $C P$ violation, is seen (the asymmetry exhibits a significant cosine term). As shown above, the first measurement already indicated $C P$ violation in decays with a significance of $2.9 \sigma$. The first evidence of direct $C P$ violation in a $B$ decay mode was reported with $3.2 \sigma$ significance in January 2004 using a sample of $152 \times 10^{6} B \bar{B}$ pairs [10]. Although this claim was not widely accepted at that time because the result of the BaBar collaboration showed a rather small $\mathscr{A}_{\pi \pi}$ value, the latest world average, $\mathscr{A}_{\pi \pi}=0.38 \pm 0.06$ [11], establishes $C P$ violation in $B^{0} \rightarrow \pi^{+} \pi^{-}$decays with a significance above $5 \sigma$.

The large direct $C P$ violation indicates that the contribution of the penguin diagram is sizable and the deviation of $\mathscr{S}_{\pi \pi}$ from $\sin 2 \phi_{2}$ may be significant. The angle $\phi_{2}$ can be extracted using the isospin relation among branching fractions and $C P$ asymmetries of $B^{0} \rightarrow \pi^{+} \pi^{-}, B^{0} \rightarrow \pi^{0} \pi^{0}$, and $B^{+} \rightarrow \pi^{+} \pi^{0}$ decays; this was first proposed by M. Gronau and D. London [12]. The result, shown in Fig. [5, is obtained using the results for $\mathscr{S}_{\pi \pi}$ and $\mathscr{A}_{\pi \pi}$ given above and the world average values of branching fractions of the three $B \rightarrow \pi \pi$ modes and direct $C P$ asymmetry in $B^{0} \rightarrow \pi^{0} \pi^{0}$. Using 

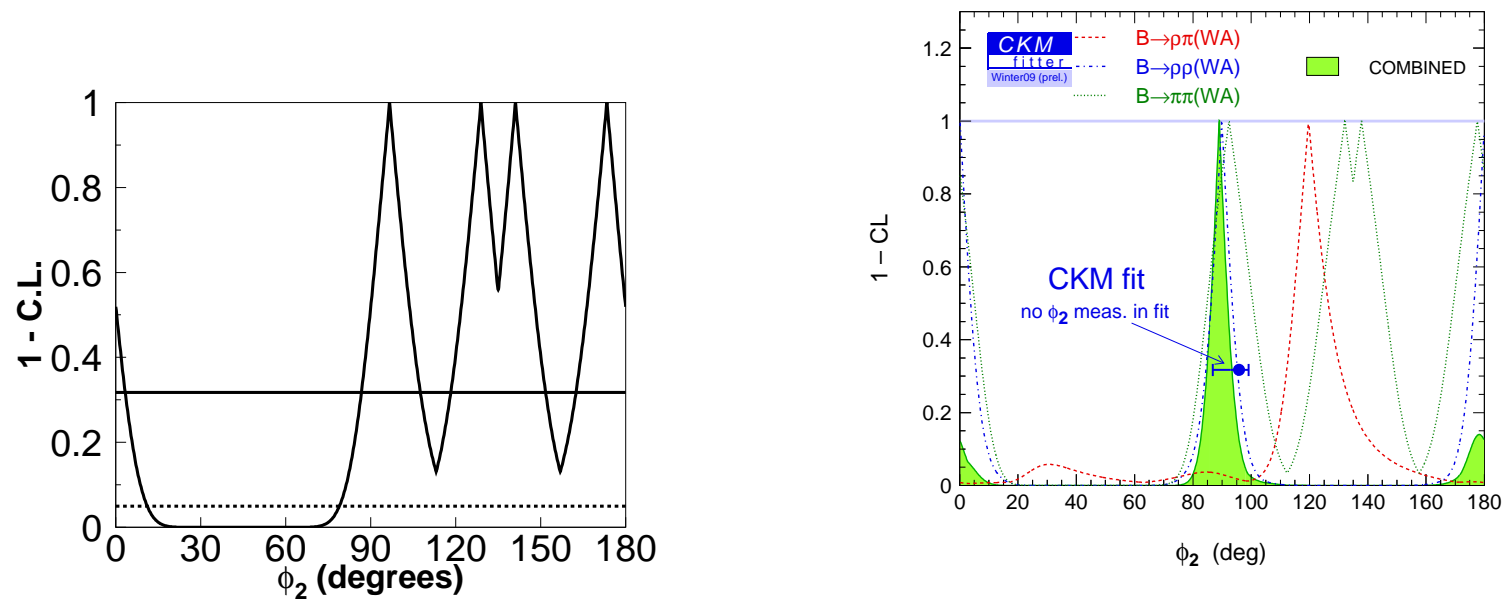

Figure 5: Left: $1-$ C.L. for a range of $\phi_{2}$ values as obtained with an isospin analysis of $B \rightarrow \pi \pi$ decays. The solid and dashed lines indicate C.L. $=68.3 \%$ and $95 \%$, respectively. Right: $1-$ C.L. as a function of $\phi_{2}$ from the average of the Belle and $\mathrm{BaBar}$ results of $B^{0} \rightarrow \pi^{+} \pi^{-}, B^{0} \rightarrow \rho \pi, B^{0} \rightarrow \rho \rho$.

this method, there are multiple discrete ambiguities for the angle $\phi_{2}$. The solution that is closest to the global fit result [13] gives $\phi_{2}=(97 \pm 11)^{\circ}$.

The final state in $B^{0} \rightarrow \rho^{+} \pi^{-}$decay is not a $C P$ eigenstate, but the decay proceeds through the same quark diagrams as $B^{0} \rightarrow \pi^{+} \pi^{-}$. Since $B^{0}$ and $\bar{B}^{0}$ can decay to $\rho^{+} \pi^{-}$, time-dependent $C P$ violation can occur and provide information on $\phi_{2}$. Here the final state is $\pi^{+} \pi^{-} \pi^{0}$ and the decay $B^{0} \rightarrow \pi^{+} \pi^{-} \pi^{0}$ contains three intermediate states; $B^{0} \rightarrow \rho^{+} \pi^{-}, \rho^{-} \pi^{+}$, and $\rho^{0} \pi^{0}$. These three amplitudes interfere and their magnitudes and relative strong phases can be extracted from a Dalitz plot amplitude analysis. Knowing the hadronic phases of these amplitudes in the Dalitz plane, a time-dependent Dalitz plot analysis allows the determination of $\phi_{2}$ [14]. This method provides $\phi_{2}$ without ambiguities (assuming large signal statistics) except for $\phi_{2} \rightarrow \phi_{2}+\pi$.

The reconstruction and continuum suppression are similar to the $B^{0} \rightarrow \pi^{+} \pi^{-}$analysis with an additional $\pi^{0}$ reconstructed in the $\pi^{0} \rightarrow \gamma \gamma$ decay mode. $C P$ violation parameters are obtained from a three-dimensional fit to the distribution of $\Delta t$ and two Dalitz distribution parameters, $M_{\pi^{+}}^{2} \pi^{0}$ and $M_{\pi^{-} \pi^{0}}^{2}$. Belle performed the analysis using $449 \times 10^{6} B \bar{B}$ pairs [15]. The amplitudes include $\rho$ (770) and higher mass resonances, $\rho(1450)$ and $\rho(1700)$. The time-dependent Dalitz plot distribution is parameterized with 27 real parameters describing the components that have different time- and Dalitz plot behaviors. $C P$ violation parameters for $B^{0} \rightarrow \rho^{ \pm} \pi^{\mp}, B^{0} \rightarrow \pi^{0} \pi^{0}$ decays and $\phi_{2}$ are extracted from these parameters. We obtain a $68^{\circ}<\phi_{2}<95^{\circ}$ at a $68.3 \%$ confidence level (C.L.) interval for the solution consistent with the global fit result. A large region $\left(0^{\circ}<\phi_{2}<5^{\circ}, 23^{\circ}<\right.$ $\phi_{2}<34^{\circ}$, and $109^{\circ}<\phi_{2}<180^{\circ}$ ) also remains. With a larger data sample, a more restrictive constraint without ambiguities is expected from this measurement.

In the $B^{0} \rightarrow \rho^{+} \rho^{-}$mode a pseudoscalar decays into two vector particles and the final state is a mixture of $C P$-even and $C P$-odd amplitudes. In order to extract the fraction of each $C P$ component, an angular analysis is required. Fortunately, the fraction of the longitudinal polarization turns out to be close to $100 \%$ [16, 17, 18], simplifying the measurement. The signal candidates are 
reconstructed in $\rho^{ \pm} \rightarrow \pi^{ \pm} \pi^{0}$ decays. Because of two $\pi^{0}$ s in the final state, the combinatorial background due to fake $\pi^{0}$ candidates is very large. The results using $535 \times 10^{6} B \bar{B}$ pairs are [19]:

$$
\begin{aligned}
& \mathscr{A}_{\rho^{+} \rho^{-}}=+0.16 \pm 0.21(\text { stat }) \pm 0.07(\text { syst }) \\
& \mathscr{S}_{\rho^{+} \rho^{-}}=+0.19 \pm 0.30(\text { stat }) \pm 0.07(\text { syst })
\end{aligned}
$$

In this mode, $\phi_{2}$ can be obtained using an isospin relation similar to that in the $B^{0} \rightarrow \pi^{+} \pi^{-}$case. Because the branching fraction for $B^{0} \rightarrow \rho^{0} \rho^{0}$ is much smaller than those of $B^{0} \rightarrow \rho^{+} \rho^{-}$and $B^{+} \rightarrow$ $\rho^{+} \rho^{0}$, the deviation of $\phi_{2}$ from the measured value is small and some ambiguities are degenerate. So far only an upper limit on $\mathscr{B}\left(B^{0} \rightarrow \rho^{0} \rho^{0}\right)$ has been obtained; this is used in the isospin analysis. The isospin analysis gives $62^{\circ} \leq \phi_{2} \leq 106^{\circ}$ at the $68.3 \%$ C.L.

All of the above results and results from the BaBar collaboration can be combined to obtain the $\phi_{2}$ constraint shown in Fig. 5[ [13]. $\phi_{2}=\left(89.0_{-4.2}^{+4.4}\right)^{\circ}$ is obtained at a $68.3 \%$ C.L.

\section{Measurement of $\phi_{3}$}

The angle $\phi_{1}$ has been now measured with high precision (Sect.2). Measurement of the angle $\phi_{2}$ is more difficult due to theoretical uncertainties from the contributions of penguin diagrams (Sect. 3). Precise determination of the third angle of the unitarity triangle, $\phi_{3}$, is possible using $B^{ \pm} \rightarrow D K^{ \pm}$decays. However, it requires much more data than determinations of the other angles. The determination of $\phi_{3}$ is theoretically clean due to the absence of loop contributions; $\phi_{3}$ can be determined using tree-level processes only, exploiting the interference between $b \rightarrow c \bar{u} d$ and $b \rightarrow u \bar{c} d$ transitions that occurs when a process involves a neutral $D$ meson reconstructed in a final state accessible to both $D^{0}$ and $\bar{D}^{0}$ decays. Therefore, $\phi_{3}$ provides an SM benchmark, and its precise measurement is crucial in order to disentangle non-SM contributions to other processes, via global CKM fits.

Several different $D$ decays have been studied in order to maximize the sensitivity to $\phi_{3}$. The archetype is the use of $D$ decays to $C P$ eigenstates, a method proposed by M. Gronau, D. London, and D. Wyler (and called the GLW method) [20]. Belle makes use of $C P$-even modes $\left(D_{1}\right)$, such as $K^{+} K^{-}$, and $C P$-odd modes $\left(D_{2}\right)$, such as $K_{S}^{0} \pi^{0}$. To extract $\phi_{3}$ using the GLW method, the following observables sensitive to $C P$ violation are used: the asymmetries

$$
\mathscr{A}_{1,2} \equiv \frac{\mathscr{B}\left(B^{-} \rightarrow D_{1,2} K^{-}\right)-\mathscr{B}\left(B^{+} \rightarrow D_{1,2} K^{+}\right)}{\mathscr{B}\left(B^{-} \rightarrow D_{1,2} K^{-}\right)+\mathscr{B}\left(B^{+} \rightarrow D_{1,2} K^{+}\right)}= \pm \frac{2 r_{B} \sin \delta_{B} \sin \phi_{3}}{1+r_{B}^{2} \pm 2 r_{B} \cos \delta_{B} \cos \phi_{3}}
$$

and the ratios

$$
\mathscr{R}_{1,2} \equiv \frac{\mathscr{B}\left(B^{-} \rightarrow D_{1,2} K^{-}\right)+\mathscr{B}\left(B^{+} \rightarrow D_{1,2} K^{+}\right)}{\mathscr{B}\left(B^{-} \rightarrow D^{0} K^{-}\right)+\mathscr{B}\left(B^{+} \rightarrow D^{0} K^{+}\right)}=1+r_{B}^{2} \pm 2 r_{B} \cos \delta_{B} \cos \phi_{3}
$$

where $r_{B}$ is the ratio of the magnitudes of the two tree diagrams shown in Fig. 6 and $\delta_{B}$ is their strong-phase difference. The value of $r_{B}$ is given by the product of the ratio of the CKM matrix elements $\left|V_{u b}^{*} V_{c s}\right| /\left|V_{c b}^{*} V_{u s}\right| \sim 0.38$ and the color suppression factor, which altogether results in a value of around 0.1. In the expressions above, mixing and $C P$ violation in the neutral $D$ meson system are neglected. Among these four observables, $\mathscr{R}_{1,2}$ and $\mathscr{A}_{1,2}$, only three are independent (since $\mathscr{A}_{1} \mathscr{R}_{1}=-\mathscr{A}_{2} \mathscr{R}_{2}$ ). Recently, Belle updated their GLW analysis using their final data sam- 

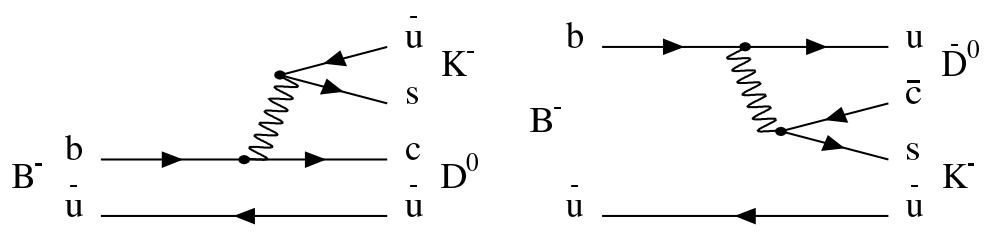

Figure 6: Feynman diagrams for $B^{-} \rightarrow D^{0} K^{-}$and $B^{-} \rightarrow \bar{D}^{0} K^{-}$.

$\mathrm{r}$

Table 4: Results of the GLW analysis for $B^{ \pm} \rightarrow D K^{ \pm}$mode.

\begin{tabular}{cc}
\hline \hline $\mathscr{R}_{1}$ & $1.03 \pm 0.07 \pm 0.03$ \\
$\mathscr{R}_{2}$ & $1.13 \pm 0.09 \pm 0.05$ \\
$\mathscr{A}_{1}$ & $+0.29 \pm 0.06 \pm 0.02$ \\
$\mathscr{A}_{2}$ & $-0.12 \pm 0.06 \pm 0.01$ \\
\hline \hline
\end{tabular}

ple of $772 \times 10^{6} B \bar{B}$ pairs [21]. The analysis uses $D^{0}$ decays to $K^{+} K^{-}$and $\pi^{+} \pi^{-}$as $C P$-even

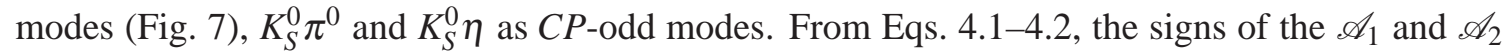
asymmetries should be opposite, which is confirmed by experiment (Table 4 ).
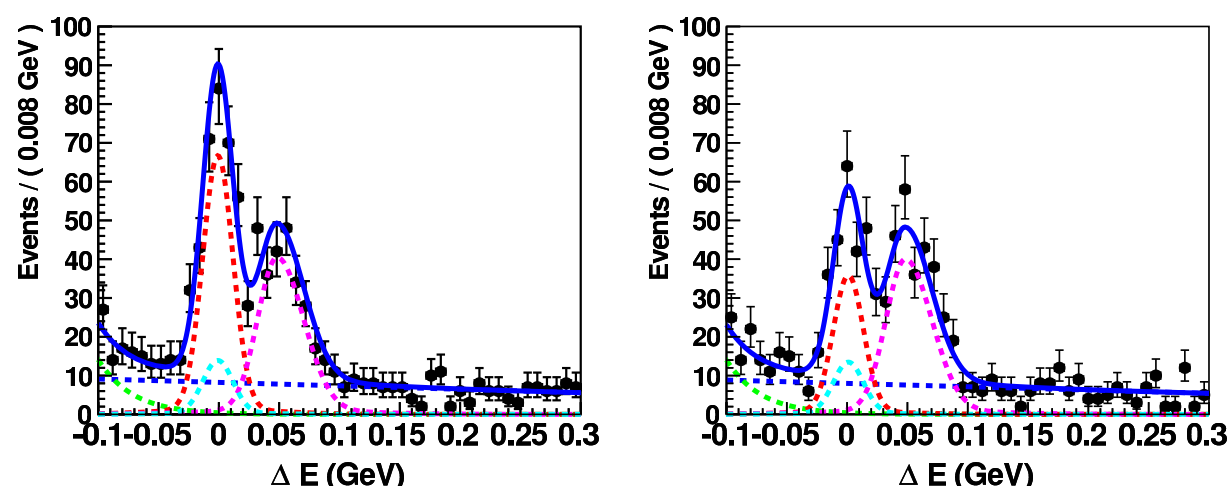

Figure 7: Signals for $B^{ \pm} \rightarrow D_{1} K^{ \pm}$decays. The left (right) figure is for $B^{-}\left(B^{+}\right)$decays. The plotted variable, $\Delta E$, peaks at zero for signal decays, while background from $B^{ \pm} \rightarrow D \pi^{ \pm}$appears as a satellite peak at positive values.

The difficulties in the application of the GLW methods arise primarily due to the small magnitude of the $C P$ asymmetry of the $B^{ \pm} \rightarrow D_{C P} K^{ \pm}$decay, which may lead to significant systematic uncertainties in the observation of the $C P$ violation. An alternative approach was proposed by D. Atwood, I. Dunietz, and A. Soni [22]. Instead of using $D^{0}$ decays to $C P$ eigenstates, the ADS method uses Cabibbo-favored and doubly Cabibbo-suppressed decays: $\bar{D}^{0} \rightarrow K^{-} \pi^{+}$and $D^{0} \rightarrow K^{-} \pi^{+}$. In the decays $B^{+} \rightarrow\left[K^{-} \pi^{+}\right]_{D} K^{+}$and $B^{-} \rightarrow\left[K^{+} \pi^{-}\right]_{D} K^{-}$, the suppressed $B$ decay is followed by a Cabibbo-allowed $D^{0}$ decay, and vice versa. Therefore, the interfering amplitudes are of similar magnitude, and one can expect a large $C P$ asymmetry. Unfortunately, the branching ra- 
tios of the decays mentioned above are small. The observable that is measured in the ADS method is the ratio of the suppressed and allowed branching fractions:

$$
\mathscr{R}_{\mathrm{ADS}}=\frac{\mathscr{B}\left(B^{ \pm} \rightarrow\left[K^{\mp} \pi^{ \pm}\right]_{D} K^{ \pm}\right)}{\mathscr{B}\left(B^{ \pm} \rightarrow\left[K^{ \pm} \pi^{\mp}\right]_{D} K^{ \pm}\right)}=r_{B}^{2}+r_{D}^{2}+2 r_{B} r_{D} \cos \phi_{3} \cos \delta
$$

and

$$
\mathscr{A}_{\mathrm{ADS}}=\frac{\mathscr{B}\left(B^{-} \rightarrow\left[K^{+} \pi^{-}\right]_{D} K^{-}\right)-\mathscr{B}\left(B^{+} \rightarrow\left[K^{-} \pi^{+}\right]_{D} K^{+}\right)}{\mathscr{B}\left(B^{-} \rightarrow\left[K^{+} \pi^{-}\right]_{D} K^{-}\right)+\mathscr{B}\left(B^{+} \rightarrow\left[K^{-} \pi^{+}\right]_{D} K^{+}\right)}=2 r_{B} r_{D} \sin \phi_{3} \sin \delta / \mathscr{R}_{\mathrm{ADS}},
$$

where $r_{D}$ is the ratio of the doubly Cabibbo-suppressed and Cabibbo-allowed $D^{0}$ decay amplitudes and $\delta$ is the sum of strong phase differences in $B$ and $D$ decays: $\delta=\delta_{B}+\delta_{D}$. The ADS analysis [23] using the full $\Upsilon(4 S)$ data sample was reported by the Belle collaboration (Fig. 8). The analysis uses $B^{ \pm} \rightarrow D K^{ \pm}$decays with $D^{0}$ decaying to $K^{+} \pi^{-}$and $K^{-} \pi^{+}$(and their charge-conjugated partners). The signal yield obtained is $56_{-14}^{+15}$ events, which corresponds to the first evidence of an ADS signal (with a significance of $4.1 \sigma$ ); the ratio of the suppressed and allowed modes is summarized in Table 5. Although the analyses with $B^{ \pm} \rightarrow D K^{ \pm}$decays give the most precise results, different $B$ decays have also been studied. The use of two additional decay modes, $D^{*} \rightarrow D \pi^{0}$ and $D^{*} \rightarrow D \gamma$, provides an extra handle on the extraction of $\phi_{3}$ from $B^{ \pm} \rightarrow D^{*} K^{ \pm}$, which is becoming visible in the most recent results [21].

Table 5: Results of the Belle ADS analyses.

\begin{tabular}{lcc}
\hline \hline Mode & $\mathscr{R}_{\mathrm{ADS}}$ & $\mathscr{A}_{\mathrm{ADS}}$ \\
\hline$B \rightarrow D K$ & $0.0163_{-0.0041-0.0013}^{+0.0044+0.0007}$ & $-0.39_{-0.28-0.03}^{+0.26+0.04}$ \\
$B \rightarrow D^{\star} K, D^{\star} \rightarrow D \pi^{0}$ & $0.010_{-0.007-0.002}^{+0.008+0.001}$ & $+0.4_{-0.7-0.1}^{+1.1+0.2}$ \\
$B \rightarrow D^{\star} K, D^{\star} \rightarrow D \gamma$ & $0.036_{-0.012}^{+0.014} \pm 0.002$ & $-0.51_{-0.29}^{+0.33} \pm 0.08$ \\
\hline \hline
\end{tabular}

A Dalitz plot analysis of a three-body $D$ meson final state allows one to obtain all the information required for determination of $\phi_{3}$ in a single decay mode. Three-body final states such as $K_{S}^{0} \pi^{+} \pi^{-}$have been suggested as promising modes [24] for the extraction of $\phi_{3}$. As in the GLW and ADS methods, the two amplitudes interfere if the $D^{0}$ and $\bar{D}^{0}$ mesons decay into the same final state $K_{S}^{0} \pi^{+} \pi^{-}$. Assuming no $C P$ asymmetry in neutral $D$ decays, the amplitude for $B^{+} \rightarrow D\left[K_{S} \pi^{+} \pi^{-}\right] K^{+}$decay as a function of Dalitz plot variables $m_{+}^{2}=m_{K_{S}^{0} \pi^{+}}^{2}$ and $m_{-}^{2}=m_{K_{S}^{0} \pi^{-}}^{2}$ is

$$
f_{B^{+}}=f_{D}\left(m_{+}^{2}, m_{-}^{2}\right)+r_{B} e^{i \phi_{3}+i \delta_{B}} f_{D}\left(m_{-}^{2}, m_{+}^{2}\right)
$$

where $f_{D}\left(m_{+}^{2}, m_{-}^{2}\right)$ is the amplitude of the $\bar{D}^{0} \rightarrow K_{S}^{0} \pi^{+} \pi^{-}$decay. Similarly, the amplitude for $B^{-} \rightarrow D\left[K_{S} \pi^{+} \pi^{-}\right] K^{-}$decay is

$$
f_{B^{-}}=f_{D}\left(m_{-}^{2}, m_{+}^{2}\right)+r_{B} e^{-i \phi_{3}+i \delta_{B}} f_{D}\left(m_{+}^{2}, m_{-}^{2}\right) .
$$

The $\bar{D}^{0} \rightarrow K_{S}^{0} \pi^{+} \pi^{-}$decay amplitude $f_{D}$ can be determined from a large sample of flavor-tagged $\bar{D}^{0} \rightarrow K_{S}^{0} \pi^{+} \pi^{-}$decays produced in continuum $e^{+} e^{-}$annihilation. Once $f_{D}$ is known, a simultaneous fit to $B^{+}$and $B^{-}$data allows the contributions of $r_{B}, \phi_{3}$ and $\delta_{B}$ to be separated. The method has 

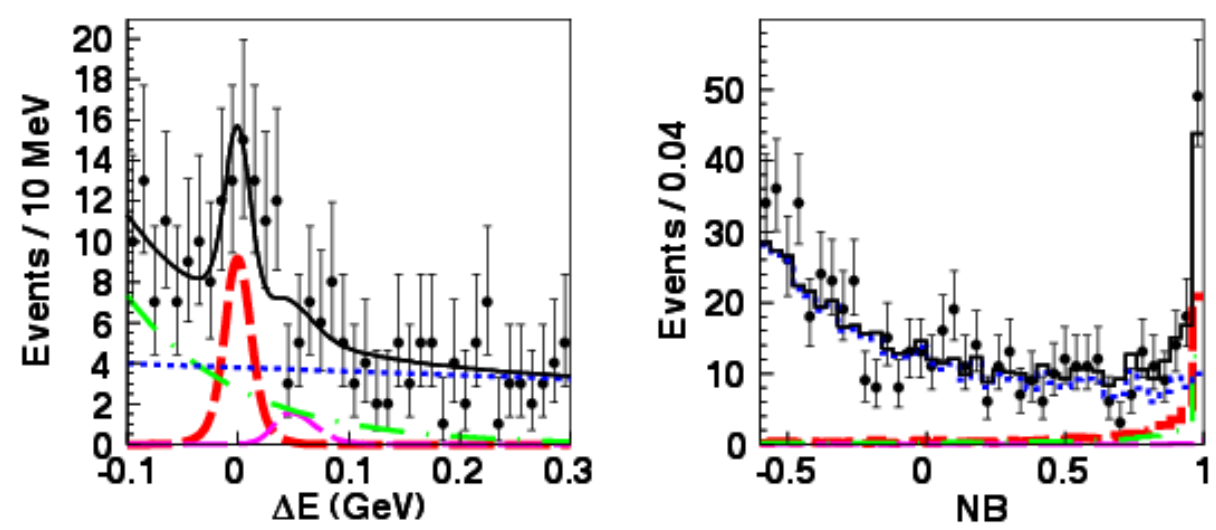

Figure 8: Signal for $B^{ \pm} \rightarrow D K^{ \pm}$decays from Belle ADS analysis. In these $\Delta E$ and $N B$ (continuum suppression variable) distributions, $\left[K^{+} \pi^{-}\right]_{D} K^{-}$components are shown by thicker dashed curves (red).

only two-fold ambiguity: $\left(\phi_{3}, \delta_{B}\right)$ and $\left(\phi_{3}+180^{\circ}, \delta_{B}+180^{\circ}\right)$ solutions cannot be distinguished. To test the consistency of the fit, the same procedure was applied to the $B^{ \pm} \rightarrow D^{(*)} \pi^{ \pm}$control samples and the $B^{ \pm} \rightarrow D^{(*)} K^{ \pm}$signal. A combined unbinned maximum likelihood fit to the $B^{+}$and $B^{-}$samples with free parameters $r_{B}, \phi_{3}$, and $\delta_{B}$ yields the values given in Table 6 . Combining $B^{ \pm} \rightarrow D K^{ \pm}$and $B^{ \pm} \rightarrow D^{*} K^{ \pm}$, we obtain [25] the value $\phi_{3}=\left(78_{-12}^{+11} \pm 4 \pm 9\right)^{\circ}$, where the sources of uncertainties are statistical, systematic, and due to imperfect knowledge of the amplitude model that describes $D \rightarrow K_{S}^{0} \pi^{+} \pi^{-}$decays. The last source of uncertainty can be eliminated by binning

Table 6: Results of Belle Dalitz plot analyses.

\begin{tabular}{lccc}
\hline \hline Mode & $\phi_{3}\left(^{\circ}\right)$ & $\delta_{B}\left(^{\circ}\right)$ & $r_{B}$ \\
\hline$B \rightarrow D K$ & $81_{-15}^{+13} \pm 5$ & $137_{-16}^{+13} \pm 4$ & $0.16 \pm 0.04 \pm 0.01$ \\
$B \rightarrow D^{\star} K$ & $74_{-20}^{+19} \pm 4$ & $342_{-21}^{+19} \pm 3$ & $0.20 \pm 0.07 \pm 0.01$ \\
\hline \hline
\end{tabular}

the Dalitz plot (Refs. [24, 26]), using information on the average strong phase difference between $D^{0}$ and $\bar{D}^{0}$ decays in each bin that can be determined using quantum correlated $\psi(3770)$ data. Results have been published recently by CLEO-c [27]. The measured strong phase difference is used to obtain a model-independent result [28]:

$$
\phi_{3}=(77 \pm 15 \pm 4 \pm 4)^{\circ} \text {, }
$$

where the last uncertainty is due to the statistical precision of the CLEO-c results.

\section{Acknowledgments}

This work is partially supported by grants of Russian Foundation for Basic Research 12-0201032 and 12-02-31363 and 14-02-31527. 


\section{References}

[1] A. Abashian et al. (Belle Collaboration), Nucl. Instrum. Meth. A 479 (2002), 117 and references therein.

[2] S. Kurokawa and E. Kikutani, Nucl. Instrum. Meth. A 499 (2003), 1.

[3] J. Beringer et al. (Particle Data Group), Phys. Rev. D 86 (2012), 010001.

[4] I. Adachi et al. (Belle Collaboration), Phys. Rev. Lett. 108 (2012), 171802.

[5] K. Abe et al. (Belle Collaboration), Phys. Rev. Lett. 91 (2003),261602.

[6] K.-F. Chen et al. (Belle Collaboration), Phys. Rev. Lett. 98 (2007), 031802.

[7] Y. Nakahama et al. (Belle Collaboration), Phys. Rev. D 82 (2010), 073011.

[8] K. Abe et al. (Belle Collaboration), Phys. Rev. Lett. 89 (2002), 071801.

[9] H. Ishino et al. (Belle Collaboration), Phys. Rev. Lett. 95 (2005), 101801.

[10] K. Abe et al. (Belle Collaboration), Phys. Rev. Lett. 93 (2004), 021601.

[11] Y. Amhis et al. (Heavy Flavor Averaging Group), arXiv:1207.1158 and online update at http://www.slac.stanford.edu/xorg/hfag.

[12] M. Gronau and D. London, Phys. Rev. Lett. 65 (1990), 3381.

[13] J. Charles et al. (CKMfitter Group), Eur. Phys. J. C41 (2005), 1.

[14] H.R. Quinn and A.E. Snyder, Phys. Rev. D 48 (1993), 2139.

[15] A. Kusaka et al. (Belle Collaboration), Phys. Rev. Lett. 98 (2007), 221602, Phys. Rev. D 77 (2008), 072001.

[16] J. Zhang et al. (Belle Collaboration), Phys. Rev. Lett. 91 (2003), 221801.

[17] A. Somov et al. (Belle Collaboration), Phys. Rev. Lett. 96 (2006), 171801.

[18] B. Aubert et al. (BaBar Collaboration), Phys. Rev. Lett. 97 (2006), 261801, Phys. Rev. D 76 (2007), 052007.

[19] A. Somov et al. (Belle Collaboration), Phys. Rev. D 76 (2007), 011104.

[20] M. Gronau, D. London, D. Wyler, Phys. Let. B 253 (1991), 483; M. Gronau, D. London, D. Wyler, Phys. Let. B 265 (1991), 172.

[21] Belle Collaboration (2011). Preliminary results presented at Lepton Photon 2011 (BELLE-CONF-1112).

[22] D. Atwood, I. Dunietz, A. Soni, Phys. Rev. Lett. 78 (1997), 3357.

[23] Y. Horii et al. (Belle Collaboration), Phys. Rev. Lett. 106 (2011), 231803.

[24] A. Giri, Yu. Grossman, A. Soffer, J. Zupan, Phys. Rev. D 68 (2003), 054018; A. Bondar. Proceedings of BINP Special Analysis Meeting on Dalitz Analysis, 24-26 Sep. 2002, unpublished.

[25] A. Poluektov et al. (Belle Collaboration), Phys. Rev. D 81 (2010), 112002.

[26] A. Bondar, A. Poluektov, Eur. Phys. J. C 47 (2006), 347; A. Bondar, A. Poluektov, Eur. Phys. J. C 55 (2008), 51.

[27] R. A. Briere et al. (CLEO Collaboration), Phys. Rev. D 80 (2009), 032002.

[28] H. Aihara et al. (Belle Collaboration), Phys. Rev. D 85 (2012), 112014. 